\title{
CORRELATION INEQUALITIES IN THE VICINITY OF THE LIQUID-GAS CRITICAL POINT
}

\author{
V.Russier, D.Di Caprio, J.P.Badiali \\ Laboratoire SRSI, Université P. et M. Curie, \\ 4 Place Jussieu 75230 Paris Cedex 05 France
}

Received September 12, 1995

\begin{abstract}
The behaviour of the many -body correlation functions in the vicinity of the liquid -gas critical point is investigated. The critical point is assumed to exist and is therefore introduced by the means of a phenomenological equation of state. We use only the framework of the liquid -state physics and thus no reference to an effective Landau Ginzburg hamiltonian is made. From the relations linking the total correlation functions $h^{(n)}$ and $h^{(n+1)}$, we show that the integrals of all the $h^{(n)}$ diverge at the critical point. We then find that the GKS inequalities are satisfied under the condition that all the distances between the particles are asymptotically large. The range of validity of these inequalities in terms of interparticle distances is given and a particular attention must be paid to the fact that usual asymptotic approximations of the liquid state theory are no more valid.
\end{abstract}

\section{Introduction}

The structure of a one component liquid is usually obtained from the n-body distribution functions, $\rho(1,2, \ldots, n)$, which give the probability of finding the particles at positions $r_{1}, r_{2}, \ldots, r_{n}$ or equivalently by the density correlation functions. Different kinds of methods are available in the fields of the liquid state theory to deal with these functions [1]. However, in the vicinity of the critical point, alternative theories have been developped: it is well known that some properties of the liquid - gas critical point (CP) can be obtained from renormalization group methods by using an effective Landau- Ginzburg hamiltonian (The usual $\Phi^{4}$ theory or Ising model) [2-4]. Moreover, from experimental results, it is well accepted that the universality class of the liquid - gas critical point is that of the three dimensional Ising model.

However, the Landau-Ginzburg hamiltonian is a functional of a field $\Phi(r)$, which, in the case of a liquid, cannot be easily related to a physical microscopic quantity. It is then clear that all the properties of the Ising model cannot be applied to the liquid -gas critical point. In particular, the correlation functions of the Ising model, which are relative to the field $\Phi$, cannot be assimilated to the density correlation functions of the liquid, even in the vicinity of the critical point. As a result, the peculiarities of the Ising model correlation functions cannot be directly transfered to the correlation functions of a fluid near the CP. For instance, in the Ising model the $(n+$ 1 ) and $n$-body correlation functions are related through the well-known 
GKS inequalities [5-7], which hold whatever the interparticle distances; it is clear that this cannot be the case for a fluid. Nevertheless, for infinitely large distances, and in the vicinity of the $\mathrm{CP}$, the correlation functions of a fluid may satisfy such inequalities. The purpose of this work is precisely to determine if this is indeed the case.

We investigate the behaviour of the usual $n$-body corelation functions of the liquid state theory in order to determine if they satisfy particular relations, in the close vicinity of the critical point; the GKS inequalities are kept in mind as a guideline. However, since we do not start from an effective field dependent hamiltonian, we have to state from the begining a phenomenological critical equation of state and the behaviour of the two -body total correlation function, the integral of which is related to the isothermal compressibility which diverges at the critical point. This amounts to assume both the existence of the critical point and that the critical exponents $\delta$ and $\eta$ are known. Then we use the integral equations given by Baxter [8] which allow to relate the $(n+1)$ and the $n$-body correlation functions. In addition, we use the $n$-body Ornstein - Zernike (OZ) equation from which we can expand the $n$-body total correlation function in terms of a finite number of Mayer graphs, involving the $h^{(2)}$ total correlation function and the $c^{(p)}$ direct correlation functions, with $3 \leq p \leq n$.

\section{Multibody correlation functions}

In a simple fluid, we can define two types of correlation functions: the total correlations, denoted by $h^{(n)}$, and the direct correlation functions denoted by $c^{(n)}$. They can be defined from functional derivative of the grand partition function, $\Xi$ with respect to the activity $z(r)$, or of the excess Helmoltz free energy, $A_{e x}$ with respect to the density $\rho(r)[1]$

$$
\begin{aligned}
\rho(1) . . \rho(n) h^{(n)} & =z(1) . . z(n)\left[\delta^{n} \ln (\Xi) / \delta z(1) . . \delta z(n)\right], \\
c^{(n)} & =-\beta\left[\delta^{n} A_{e x} / \delta \rho(1) . . \delta \rho(n)\right] .
\end{aligned}
$$

In $(2.1,2.2), f^{(n)}$ means $f^{(n)}(1,2, . ., n)(f=h$ or $c)$.

For our analysis, alternative and equivalent definitions in terms of Mayer diagrams are useful. $h^{(n)}$ is the sum of all simple diagrams with $n$ white circles, black $\rho$ circles and $f$ bonds, where $f$ is the Mayer function; $c^{(n)}$ is the sum of all the same kind of diagrams which are free of nodal points, which means that they are at least doubly connected.

Then, the $n$-body distribution functions are obtained from the total correlation functions

$$
g^{(n)}=1+\sum_{Q}\left[\Pi\left[h^{(m)}\left(i_{1}, i_{2}, . ., i_{m}\right)\right]\right],
$$

where the sum runs over all the partitions $Q$ of the set $(1,2, . ., n)$ in distinct and non -overlapping subsets $\left(i_{1}, i_{2}, . ., i_{m}\right)$ with $1 \leq m \leq n, i_{p} \in(1,2, . ., n)$ and the product runs over all the subsets of the particles under consideration. When the subset includes only one point, the corresponding $h$ function is set equal to 1 . With these notations, the so-called GKS inequalities read

$$
g^{(n+p)}-g^{(n)} g^{(p)} \geq 0
$$


and one can convince oneself easily that the liquid correlation functions satisfy (2.4) if the two following conditions are fulfilled

$$
\begin{aligned}
& h^{(2)} \geq 0, \\
& h^{(p)}(n+1, i, . .) \ll h^{(2)}(n+1, i) .
\end{aligned}
$$

In the following we investigate if these equation hold.

\section{Characterization of the critical point}

First we state the existence and the characteristics of the critical point. We consider the critical isotherm, and thus the deviation $\Delta \rho=\rho-\rho_{c}$ where $\rho$ is the density and $\rho_{c}$ its critical value measures the distance to the critical point. The phenomenological equation of state relating the pressure $P$ to $\Delta \rho$ is the following $[2]$

$$
\Delta P=P-P_{c}=(\Delta \rho)^{\delta} .
$$

We assume that the critical exponents are known and satisfy the socalled scaling relations; in particular we have: $\delta=(d+2-\eta) /(d-2+\eta)$. Finally, we assume that the two body correlation function presents a long range part of the usual form [9]

$$
h(r)=\frac{A}{r^{d-2+\eta}} \exp (-r / \xi) .
$$

The isothermal compressibility $\chi$ is related to the integral of the two -body total correlation function and of the two -body direct correlation function

$$
\rho \chi / \beta=1+\rho \int h^{(2)}=\left(1-\rho \int c^{(2)}\right)^{-1} .
$$

Now, from the thermodynamic stability condition $\chi>0$, and because we expect $h^{(2)}$ to be monotonous for large distances (only the correlation length, $\xi$ is relevant for the long range part of the correlation functions), we can conclude that the first line of (2.5) is satisfied; in other words, for large distances, $h^{(2)}>0$ and therefore the GKS inequality at order 2 is satisfied. Furthermore, from (3.6) and the definition of $\chi, \beta / \rho \chi=\beta \partial P / \partial \rho$ we deduce the way in which the integral of $h^{(2)}$, which we denote by $H^{(2)}$, diverges when the $\mathrm{CP}$ is approached.

$$
H^{(2)}=\int h^{(2)} \sim \Delta \rho^{(1-\delta)}
$$

similarly, from (3.7) we obtain the behaviour of the correlation length with respect to $\Delta \rho$

$$
\xi=\Delta \rho^{-1 / d_{\Phi}}, \quad \quad d_{\Phi}=(d-2+\eta) / 2 .
$$




\section{Determination of an upper bound for $h^{(n)}$}

\subsection{Baxter relations and consequences}

In order to investigate (2.5), we need a hierarchical relation between the different multi -body correlation functions. Our natural variable is $\Delta \rho$, and thus we use the equations derived first in [10], in the form given by Baxter [8] which relate the derivative of $h^{(n+1)}$ with respect to the density to $h^{(n)}$. Notice that in [11], where the asymptotic form of $h^{(3)}$ in the vicinity of the $\mathrm{CP}$ was investigated, the same set of equations was used. We have

$$
\int h^{(n+1)} d(n+1)=\left(\partial h^{(n)} / \partial \rho\right)\left(1+\rho \int h^{(2)} d 2\right)+n h^{(n)} \int h^{(2)} d 2 .(4
$$

On the other hand, the direct correlation functions satisfy

$$
\int c^{(n+1)} d(n+1)=\left(\partial c^{(n)} / \partial \rho\right) .
$$

To each function $h^{(n)}\left(\right.$ or $\left.c^{(n)}\right)$, we associate the integral $H^{(n)}\left(\right.$ or $\left.C^{(n)}\right)$ over $(n-1)$ space coordinates; because of the translational invariance, $H^{(n)}$ and $C^{(n)}$ are constants.

$$
H^{(n)}=\int h^{(n)} d 1 . . d(n-1), \quad C^{(n)}=\int c^{(n)} d 1 . . d(n-1)(.4 .
$$

From $(4.11,4.12)$, we get a hierarchical equation for both the $H^{(n)}$ and the $C^{(n)}$ sets of density dependent functions

$$
\begin{aligned}
H^{(n+1)} & =\left(1+\rho H^{(2)}\right)\left(\partial H^{(n)} / \partial \rho\right)+n H^{(2)} H^{(n)}, \\
C^{(n+1)} & =\left(\partial C^{(n)} / \partial \rho\right) .
\end{aligned}
$$

We examine the form taken by (4.14) in the vicinity of the CP; we emphasize that we focus only on the leading contributions of each quantity considered. By using (3.9) and the second equality of (3.8) we get

$$
\begin{aligned}
H^{(n)} \sim A(n) \Delta \rho^{\left(x_{n}\right)}, \quad x_{n}= & (1-(n-1) \delta) \\
A(2) & =A_{c}>0 \\
A(n) & =(-1)^{n}\left(A_{c}^{(n-1)}\left|x_{2} x_{3} . . x_{n-1}\right|\right) \\
\rho^{(n-1)} C^{(n)}=(-1)^{n}(n-2) ! & -C_{c} \Delta \rho^{\delta+1-n}
\end{aligned}
$$

In (4.16), $A(2)>0$ is a consequence of the requirement that the compressibility must be positive. We note that all the $H^{(n)}$ diverge and that their signs are alternate. Moreover, one easily shows that the Kirkwood superposition approximation overestimates $H^{(n)}$ by a factor $\Delta \rho^{(n-2)}$. On the other hand, the $C^{(n)}$ diverge at the CP only for $n>(\delta+1)$; the integrals $C^{(n)}$ include a constant term, which cannot be produced by a long range contribution to the function $c^{(n)}$, since it does not "see" the correlation length $\xi$. Therefore we isolate in $c^{(n)}$ a localized contribution, $c_{0}^{(n)}$, the integral of which is given by the first term in (4.16); since we are interested 
here only in very large distances, $c_{0}^{(n)}$ is a product of $\delta$ functions. We thus define $c_{0}^{(n)}$ and $c_{1}^{(n)}$ according to

$$
\begin{aligned}
c^{(n)} & =c_{0}^{(n)}+c_{1}^{(n)}, \\
& =\left[\rho^{(n-1)}(-1)^{n}(n-2) !\right] \delta(1-2) . . \delta(1-n)+c_{1}^{(n)}(4.17)
\end{aligned}
$$

Concerning $h^{(n)}$, in addition to the integral given in (4.16), we consider the integral of $h^{(n)}(1,2, . ., n)$ over the $(n-2)$ space coordinates, say $(3, . ., n)$ in all space; the result is then a function of the distance $r_{12}$. It is easy to see from (4.11) that, in the vicinity of the CP, this function, that we denote by $\tilde{H}^{(n)}(1,2)$ is obtained by the application of the operator:

$$
\left[H^{(2)}(\partial+(n-1)) H^{(2)}(\partial+(n-2)) . . H^{(2)}(\partial+2)\right],
$$

where $\partial(f)$ is a shortened notation for $\rho \partial(f) / \partial \rho$, on the two-body total correlation function $h^{(2)}\left(r_{12}\right)$. Since $H^{(2)}$ diverges as the CP is approached, the contribution $\partial$ in each of the $(n-3)$ first factors $(\partial+i)$ dominates. We have shown [12] that this holds when $H^{(2)}$ represents the integral of $h^{(2)}$ over a volume of characteristic length $l$ with $l / \xi \geq \xi^{-d_{\Phi}}$; thus, in the following, a large distance is to be understood as a distance $l \geq \xi^{\left(1-d_{\Phi}\right)}$. We then get the result

$$
\tilde{H}^{(n)}(1,2)=H^{(n-1)}\left(\rho \partial h^{(2)}\left(r_{12}\right) / \partial \rho+2 h^{(2)}\left(r_{12}\right)\right) .
$$

From this, we conclude that if we integrate $h^{(n)}$ over $(n-2)$ coordinates in all space and then over, say $\mathbf{r}_{2}$ in a small volume, $r_{12}<\lambda \ll \xi$, we get

$$
\begin{aligned}
H_{\lambda}^{(n)} & =\int_{r_{12}<\lambda} d 2 \int h^{(n)} d 3 . . d(n-1) \ll H^{(n)}, \\
H_{\sigma}^{(n)} & \sim H^{(n-1)},
\end{aligned}
$$

where, in (4.21), « 1 means of order $\Delta \rho^{(x>0)}$, and $\sigma$ is the molecular length. This result can be generalized to more than one "small" volume of integration, and we conclude that the leading contribution of the integral of $h^{(n)}$ is due to the region of space where all the distances involved in the set $\left(r_{i j}\right),(i, j \in(1, . ., n))$ are asymptotically large, namely are comparable to $\xi$. Thus, the integral $H^{(n)}$, is indicative of the behaviour of the $h^{(n)}$ in the case where all the distances are on the one hand of order $\xi$, and on the other hand are of the same order of magnitude. We can therefore split $h^{(n)}$ into three contributions, $h_{\sigma}^{(n)}, h_{\sigma \xi}^{(n)}, h_{\xi}^{(n)}$ corresponding to the case where all the distances are of order $\sigma$; where at least one distance is of order $\sigma$ and one distance is of order $\xi$; and where all the distances are of order $\xi$ respectively. Given the discussion above the integral of $h_{\xi}^{(n)}$ coincides with $H^{(n)}$ given in (4.16). In the following we investigate only $h_{\xi}^{(n)}$ which we write in the form

$$
h_{\xi}^{(n)}=\Sigma\left(\Pi\left(r_{i j}\right)^{-z(n)}\right) \Phi^{(n)} F\left(r_{i j} / r_{k l}\right) .
$$

The first term is the sum of the products of inverse powers of distances; the second term is a cutoff function which vanishes when one distance is 
larger than $\xi$; and the third one, in agreement with [13], is a dimensionless function of the ratio of distances. This last term describes the "angular" structure of $h^{(n)}$ : it is expected to play a central role when two length scales, $\sigma \ll \lambda_{1} \ll \lambda_{2} \leq \xi$ have to be considered in the set $\left(r_{i j}\right),(i, j \in(1, . ., n))$. When all the distances are of the same order of magnitude, then we have $F=1$. We emphasize that the function $F$ is dimensionless, and therefore plays no role concerning the "dimension" of $h^{(n)}$, which is determined by the factor $\left(\Pi\left(r_{i j}\right)^{(-z(n)}\right)$. From (4.16) we get $\Sigma_{i, j}(z(n))=n d_{\Phi}$. In the following, we focus only on $h_{\xi}^{(n)}$, and for the sake of simplicity we forget the subscript $\xi$.

\subsection{Expansion of $h^{(n)}$ in terms of Mayer graphs}

By using the $n$-body version of the Ornstein - Zernike equation, we can write down the diagrams entering in the expansion of $h^{(n)}$ from those entering in the expansion of $h^{(n-1)}$. Then we can deduce the diagrammatic expansion of $h^{(n)}$ in terms of diagrams built from $h^{(2)}$ and $c^{(m)}$ functions, $m \leq n$. The details are given in [12]. The important point is that the expansion of $h^{(n)}$ includes only a finite number of graphs, avoiding convergence problems. Then, since the superposition approximation leads to an underestimation for the dimension of $h^{(n)}$, we drop the corresponding graphs; similarly, we can drop the graphs where the root points (corresponding to the space variable $\left.r_{1}, r_{2}, . . r_{n}\right)$ do not end a $h^{(2)}$ bond. We introduce the nodes $V^{(p)}$, as the subgraphs made of convolutions of direct correlation functions, $c^{(m)}, m \leq p$, and including $p$ external points. We have thus to take into account the graphs including $h^{(2)}$ bonds, nodes $V^{(p)}$ with $3 \leq p \leq n$, where each root point ends a $h^{(2)}$ bond.

The next step consists in analysing the contribution to the nodes of the localized part of the $c^{(m)}$, as defined in (4.17). The result is that this contribution vanishes exactly and we have to take into account only the dimensioned part of the functions $c^{(m)}, c_{1}^{(m)}$ in the nodes $V^{(p)}$. This is a crucial point of the derivation, and the details can be found in [12]. It is then easy to find that the leading contribution to the nodes $V^{(p)}$ is simply $c_{1}^{(p)}$. At this stage, in order to estimate $h^{(n)}$, we have to take into account the graphs including $h^{(2)}$ bonds, $c_{1}^{(p)}$ functions as nodes, with $3 \leq p \leq n$, and where all the root points end a $h^{(2)}$ bond. In the cases where the configuration of the points $(1,2, \ldots, n)$ involves only one length scale (i.e. when $F=1)$, the estimation of $h^{(n)}$ is quite easy and in fact may be obtained simply from its dimension; nevertheless, it is important to note that in this case, the precise configuration of the nodes involved in the graphs does not play any role and we can use the graphs which include only the simplest node, namely, $c_{1}^{(3)}$. The analysis of these graphs is very simple.

The final step is to consider the case where two length scales are involved in the set $(1,2, . ., n)$. These are denoted by $\alpha$ and $\lambda$ and we consider the case: $\sigma \ll \alpha \ll \lambda \leq \xi$, which means that both $\alpha$ and $\lambda$ may be compared to $\xi, \alpha / \lambda$ takes a very small value, while $\sigma / \xi \rightarrow 0$. We separate the $n$ points into $m$ groups, $i$ of $m(i)$ points located in a volume $\alpha^{d}$ centered at $\mathbf{R}_{i}$. The coordinates of the points in the group $i$ are $\mathbf{r}_{k}=\mathbf{R}_{i}+\mathbf{r}_{i k}$ with $\left|\mathbf{r}_{i k}\right| \leq \alpha$ and the distances between the different groups are $R_{i j} \approx \lambda$. Because the graphs of the direct correlations are at least doubly connected, it is easy to show, although tedious, that the leading graphs are those in which no node 
is shared by two different groups of localized particles. Then we get for $h^{(n)}$ a clustering property: $h^{(n)}$ is a convolution product of a graph $G_{i}^{(m(i))}$ per group $i$ with the long range part of $h^{(m)}\left(R_{i j} \sim \lambda\right)$, connecting all the groups together

$$
h^{(n)} \sim h^{(m)}(\lambda) *\left\{G_{i}(\alpha)\right\}_{i=1, m} .
$$

By definition of the two length scales, $\lambda$ is the largest distance between particles in the set $(1,2, \ldots, n): \lambda=r_{\max }$. Now, from the structure of the graphs, leading to the preceding estimation of the leading contribution of $h^{(n)}$, we know that only one length scale is involved in each sub -graph $G_{i}$; we can therefore estimate $G_{i}$ from its dimension. From our analysis, we deduce the topological form of the graphs of $G_{i}$ : they are graphs representative of the leading contribution to $h_{\xi}^{(m(i)+1)}$, amputated of one $h^{(2)}$ bond. We therefore get the following estimation of $h_{\xi}^{(n)}$ in our case

$$
h_{\xi}^{(n)} \sim h^{(m)}\left(r_{\max }\right)\left[\alpha^{-(n-m) d_{\Phi}}\right] .
$$

Hence, as we are interested only in very large distances, when $n>m$, we get $h_{\xi}^{(n)} \ll h^{(m)}\left(r_{\max }\right)$. Here, $h^{(m)}\left(r_{\max }\right)$ involves now only one length scale, and thus may be estimated from its dimension, with the result: $h^{(m)}\left(r_{\max }\right) \ll h^{(2)}\left(r_{\max }\right)$. We therefore conclude that $(2.5)$ is indeed satisfied. However, our main result here, is (4.23), or (4.24), which is a stronger majoration of $h^{(n)}$ than (2.5).

\section{Conclusion}

In this work, we do not refer to an effective Landau -Ginzburg hamiltonian, and as a result we deal with the actual spacial correlations of density in the fluid.

We emphasize that it has been necessary to introduce two different length scales, and thus, we go beyond a dimensional analysis. Our important result is the clustering property (4.23), which leads to an equivalent, for the fluid, of the GKS inequalities.

Concerning the GKS inequalities, the difference with the Ising model (or the $\Phi^{4}$ theory), is that (2.5) is satisfied only in the vicinity of the CP and for asymptotically large distances. The range of validity of (2.5), and of (4.24) is: $r_{i j} \geq \xi(\sigma / \xi)^{d_{\Phi}}, i, j \in(1,2, . ., n)$. Notice that in [11] the asymptotic form of the correlation $g^{(3)}(1,2,3)-g^{(2)}(1,2)$ was investigated in the case where $r_{12}$ is of order $\sigma$ (as a consequence of the utilization of the YBG hierarchy equations) and the sign of the result is not determined as it depends on terms characteristic of the molecular scale. Thus, in that latter case, the GKS inequalities are not satisfied at order 3 even when $r_{13} \rightarrow \infty$. Finally, we mention that one can generalize this failure of the GKS inequalities to all cases where only one distance is asymptotically large in the set $\left(r_{i j}\right), i, j \in$ $(1,2, . . n)$; that is when all the particles but one are enclosed in a volume of molecular size, the $n$-th particle being a distance comparable to $\xi$ away from this volume. Such a situation cannot be represented in the framework of the Ising model, since the size of the lattice is already very large compared to the fluid molecular length. Thus, the present work illustrates one limitation of the Ising model for the modelisation of the liquid -gas critical point. 


\title{
References
}

[1] Hansen J.P., McDonald I.R. Theory of simple liquids. New-York, Academic Press, 1986.

[2] Ma S.K. Modern theory of critical phenomena. Reading, Benjamin, 1976.

[3] Brézin E., Le Guillou J.C., Zinn-Justin J. Phase transition and critical phenomena. Eds. C.Domb and M.S.Green, New-York, Academic Press, 1976, Vol. 6, p. 125-247.

[4] Zinn-Justin J. Quantum field theory and critical phenomena. Oxford, Clarendon Press, 1989.

[5] Griffiths R.B. Correlations in Ising ferromagnets. // J. Math. Phys., 1967, vol. 8, No 3, p. 478-489; Kelly D.G., Sherman S. General Griffiths' inequalities on correlation in Ising ferromagnets. // J. Math. Phys., 1968, vol. 9, No 3, p. 466-484.

[6] Baker G.A. Quantitative theory of critical phenomena. New-York, Academic Press, 1990.

[7] Glimm J., Jaffe A. Quantum physics: a functional integral point of view. New-York, Springer-Verlag, 1981.

[8] Baxter R.J. Direct correlations and their derivatives with respect to the particle density. // J. Chem. Phys., 1964, vol. 41, No 2, p. 553-558.

[9] Fisher M. Correlation functions and the critical region of simple fluids. // J. Math. Phys., 1964, vol. 5, No 7, p. 944-961.

[10] Lebowitz J.L., Percus J.K. Long range correlations in a closed system with applications to nonuniform fluids. // Phys. Rev., 1961, vol. 122, No 6, p. 1675-1691; Lebowitz J.L., Percus J.K. Statistical theory of nonuniform fluids. // J. Math. Phys., 1963, vol. 4, No 1, p. 116-123.

[11] Fisher I.Z. Long range three particle correlation at the critical point. // Sov. Phys. JETP, 1972, vol. 35, No 4, p. 811-815.

[12] DiCaprio D., Russier V., Badiali J.P. Properties of the n-body correlation functions near the liquid-gas critical point. // J.Stat. Phys., 1995, vol. 80, No 5/6, p. 1241-1278.

[13] Cardy J.L. Phase transition and critical phenomena. Eds. C.Domb and J.L.Lebowitz, New-York, Academic Press, 1987, vol. 11, p. 55-126.

\section{КОРЕЛЯЦІЙНІ НЕРІВНОСТІ В ОКОЛІ КРИТИЧНОЇ ТОЧКИ РІДИНА-ГАЗ}

\author{
В.Русьє, Д. ді Капріо, Ж.П.Бадіалі
}

Досліджуються властивості багаточастинкових кореляційних функцій в околі критичної точки рідина-газ. Вважається, що критична точка існує і тому вводиться через феноменологічне рівняння стану. Застосовуються лише методи фізики рідин, через те не має покликань на ефективний гамільтоніан Гінзбурга-Ландау. 3 рекурентних співвідношень, що пов'язують повні кореляційні функції $h^{(n)}$ та $h^{(n+1)}$ показується, що інтеграли всіх $h^{(n)}$ розбігаються в критичній точці. Після цього показується, що нерівності ГКШ задовільняються за умови, що всі відстані між частинками $є$ асимптотично великими. Область достовірності цих нерівностей вказується у термінах міжчастинкових відстаней, окрема увага звертається на той факт, що звичайні асимптотичні наближення теорії рідкого стану при цьому вже не є застосовними. 